\title{
Exponential Differences
}

\author{
By L. M. Delves
}

Abstract. The concept of functional differences is described, and the calculus of functional differences developed for the particular case of the exponential function.

1. Introduction. The usual theory of finite difference operators on a uniform mesh in one dimension proceeds in general through the following steps.

(i) Given an operator $F$ and a function $g(x)$, both defined on the space $x$, we require to form an approximation to $F g(x)$.

(ii) We expand $F$ in terms of the forward difference operator $\Delta$

$$
\begin{aligned}
\Delta f(x) & =f(x+h)-f(x), \\
F & =\sum_{n=0}^{\infty} b_{n} \Delta^{n} .
\end{aligned}
$$

(iii) We write a formal expansion for $F g$ :

$$
F g(x)=\sum_{n=0}^{\infty} b_{n} \Delta^{n} g(x)
$$

(iv) We assume there exists a strongly convergent expansion of the form

$$
g(x)=\sum_{m=0}^{\infty} a_{m} x^{m}
$$

(v) We cut off the expansion (1.3) at the term $m=M$ by assuming

$$
a_{m} \simeq 0, \quad m>M,
$$

and use the annihilation property of the difference operator $\Delta$ with respect to the set of functions $\left\{x^{p}\right\}$ :

$$
\Delta^{q} x^{p}=0, \quad q>p,
$$

to write approximately

$$
F g(x) \approx \sum_{n=0}^{M} b_{n} \Delta^{n} g(x)
$$

The accuracy of a formula such as (1.5) for given $M$ depends on the form of the function $g(x)$ through the assumption (1.3a); for a function $g(x)$ with a rapidly convergent power series expansion in $x$ over the necessary range, (1.5) may represent a good approximation, while functions whose power series expansions converge only slowly or not at all, may give complete nonsense when substituted into (1.5). In this case, the power series expansion (1.3) is clearly not appropriate.

It may well be however, that a rapidly convergent expansion in powers of some other parameter $h$ exists. If we write $h$ as a function of $x$ :

$$
h=h(x),
$$

Received December 1, 1965. Revised September 16, 1965. 
then we can use the formalism of (1.1)-(1.5) by making everywhere the change of variable

$$
y=h(x)
$$

and expanding in powers of $y$, with finite difference operators defined over a uniform mesh in $y$-space. This process of an analytic change of variables is seldom convenient, and often introduces extraneous difficulties of its own (when, for instance, a different change of variables is required over different regions of space). We give below an alternative method of procedure which retains a uniform mesh in $x$-space, and instead adapts the finite difference procedure to expansions in functions other than polynomials in $x$, by a suitable redefinition of the operator $\Delta$. The general method and its aims are discussed in Section 2, while a particular case of the method is developed in the succeeding sections, for which the function $h(x)$ of Eq. (1.6) is the exponential function

$$
h(x)=e^{-x} \text {. }
$$

2. Functional Difference Operators. We define the functional difference operator $\Delta_{f}$ with respect to the function $f(x)$, and over a mesh of width $h$, by the relation

$$
\Delta_{f} g(x)=f(x)[g(x+h)-g(x)]
$$

and the weighted shift operator $E_{f}$ by the relation

$$
E_{f} g(x)=f(x) g(x+h) \text {. }
$$

In Eqs. (2.1), (2.2), $g(x)$ is an arbitrary function of $x$. The operators $E_{f}, \Delta_{f}$ obey the operator relation

$$
\Delta_{f}=E_{f}-f(x) \text {. }
$$

Unlike the unweighted operators $E, \Delta$, they do not commute, but satisfy the commutation relation

$$
\left[E_{f}, \Delta_{f}\right] \equiv E_{f} \Delta_{f}-\Delta_{f} E_{f}=\left\{\frac{1}{f} \Delta_{f} f(x)\right\} E_{f} .
$$

We can see the relation between the operators $E_{f}, \Delta_{f}$ and a change in independent variable $x$ by taking the limit $h \rightarrow 0$. In this limit we have

$$
\Delta_{f}=h D_{f}
$$

where $D_{f}$ is the differential operator

$$
\begin{aligned}
D_{f} & =f(x) \frac{d}{d x}=\frac{d}{d y}, \\
y & =\bar{f}(x), \\
\frac{d \bar{f}(x)}{d x} & =\frac{1}{f(x)} .
\end{aligned}
$$

Hence the operator $\Delta_{f}$ has some of the properties of the unweighted operator $\Delta$ over a uniform mesh in the independent variable $y$ defined in Eq. (2.6). The correspondence is by no means complete, however. 
For our purposes the most important characteristic of $\Delta_{f}$ is the class of functions annihilated by $\left(\Delta_{f}\right)^{m}$. The relation

$$
\left(\Delta_{f}\right)^{m} g_{n}(x)=0, \quad m>n,
$$

defines a set of functions $\left\{g_{n}\right\}, n=0,1,2, \cdots$. This set is essentially uniquely defined, apart from multiplicative constants (periodics), and for any function $f(x)$ can be generated recursively from the linear difference equation

$$
\Delta_{f} g_{n}(x) \equiv f(x) \Delta g_{n}(x)=C_{n} g_{n-1}(x),
$$

where $C_{n}$ is an arbitrary periodic of period $h$. For instance, we have

$$
\begin{aligned}
g_{0}(x) & =C_{0}, \\
g_{1}(n h) & =C_{1} \sum_{m=1}^{n-1} \frac{C_{0}}{f(m h)} .
\end{aligned}
$$

For sufficiently regular $f(x)$ the set of functions $\left\{g_{n}\right\}$ is complete, and therefore for an arbitrary function $b(x)$ we can write, at least formally, expansions of the types (1.1) $-(1.5)$ in terms of $\Delta_{f}, g_{n}$ rather than $\Delta, x^{n}$ :

$$
\begin{gathered}
F=\sum_{n=0}^{\infty} b_{n} \Delta_{f}{ }^{n}, \\
h(x)=\sum_{m=0}^{\infty} a_{m} g_{m}(x), \\
F h(x) \simeq \sum_{n=0}^{\infty} b_{n} \Delta_{f}{ }^{n} h(x) .
\end{gathered}
$$

For the class of functions for which $(2.10 \mathrm{~b})$ converges rapidly, the expansion $(2.10 \mathrm{c})$ will be preferable to (1.5).

Difference operators of the form (2.1) have been considered by Levy and Lessman [1], who chose

$$
f(x)=x .
$$

The criterion (2.6) shows that this choice is (roughly) equivalent to the scale change

$$
y=\log x .
$$

However, Levy and Lessman were interested in the solution of nonlinear difference equations, rather than in expansions of the form (2.10).

The exponential differences discussed in this paper have also been introduced by a number of authors [3], [4], [5]. In particular, the interpolation formula (6.1) has been given previously by Gould [5]. I am grateful to the referee for pointing out these references.

3. Exponential Differences. We shall discuss in this paper the difference operator generated by the following choice of the function $f(x)$

$$
f(x)=e^{x}
$$

This choice has several motivations. 
(1) It leads to the particularly simple set of annihilation functions $\left\{g_{n}\right\}=\left\{e^{-n x}\right\}$. Hence, formulae of the type (2.10c) which we shall derive are exact for polynomials in $e^{-x}$ of degree less than or equal to $M$. The relationship of this result to the usual finite difference formulae, exact for finite polynomials in $x$, is apparent and very convenient.

(2) The integration rules derived in Section 7 are translationally invariant; that is, a rule defined on a region $\overline{0} x$ remains valid (and fits the same set of functions) over the translated region $\overline{t t+x}$. This property is convenient for the generation of cytolic rules by the addition of simple rules over small regions; the present work in fact arose from a general investigation of translationally invariant integration rules.

Let us define the finite difference operator $P$ by the relation

$$
\begin{aligned}
P & =-e^{x} \Delta, \\
P g(x) & =-e^{x}[g(x+h)-g(x)] .
\end{aligned}
$$

The operator $h^{-1} P$ is clearly a first order approximation to the exponential differential operator $R$ :

$$
R=-e^{x} d / d x=d / d\left(e^{-x}\right) .
$$

Both the operators $P$ and $R$ annihilate the set of functions $\left\{e^{-m x}\right\}$. We have the relations:

$$
\begin{aligned}
& P e^{-m x}=\left(1-z^{m}\right) e^{-(m-1) x} \\
& R e^{-m x}=m e^{-(m-1) x}
\end{aligned}
$$

where

$$
z=e^{-h}
$$

and hence the annihilation relations

$$
\begin{aligned}
& P^{n} e^{-m x}=0, \\
& R^{n} e^{-m x}=0,
\end{aligned}
$$

We can therefore identify the set of annihilation functions $\left\{g_{n}\right\}$, Eq. (2.7), with $\left\{e^{-n x}\right\}$.

In the following sections we derive a number of operator expansions in terms of $P$ and $R$.

4. Taylor Series Expansion. Using the simple relations

$$
\begin{aligned}
\int e^{-x} R f(x) d x & =-f(x)+C \\
\int e^{-x} u R v d x & =-u v-\int e^{-x} v R u d v,
\end{aligned}
$$

we derive in the standard manner the Taylor series expansion for a function $f(x)$ :

$$
f(b)=\sum_{n=0}^{N} \frac{\left(e^{-b}-e^{-a}\right)^{n}}{n !} R^{n} f(a)+(-1)^{N+1} \int_{a}^{b}\left[R^{N+1} f(x)\right]\left(e^{-x}-e^{-b}\right)^{N} e^{-x} d x
$$


5. An Expansion for $R$ in Terms of $P$. Let us assume that there exists an expansion of the form

$$
R=\sum_{n=0}^{\infty} a_{n} e^{-(n-1) x} P^{n} .
$$

Given such an expansion, we can find the coefficients $a_{n}$ most easily with the use of the annihilation functions $\left\{e^{-n x}\right\}$. These functions form a complete set; moreover, the operator $R$ is linear, and hence the condition (3.4b) for $m=0,1, \cdots, \infty$ is sufficient to define the coefficients $a_{n}$. Moreover, (3.5) implies that the set of linear equations obtained is triangular, and can be solved by successive substitution. The result is the expansion

$$
R=\sum_{n=1}^{\infty}\left(1-z^{n}\right)^{-1} e^{-(n-1) x} P^{n}
$$

where $z$ is defined by (3.4c). Eq. (5.2) is most easily proven by operating with both sides on the function $e^{-m x}$. The relation

$$
P^{n} e^{-m x}=\left(1-z^{m}\right)\left(1-z^{m-1}\right) \cdots\left(1-z^{m-n+1}\right) e^{-(m-n) x},
$$

then yields the identity

$$
\sum_{n=1}^{\infty}\left(1-z^{n}\right)^{-1}\left(1-z^{m}\right)\left(1-z^{m-1}\right) \cdots\left(1-z^{m-n+1}\right)=m
$$

valid for arbitrary $m$. The identity (5.4) can be proved simply for integer $m$, for which the series terminates, by induction on $m$.

6. An Interpolation Formula. In a similar manner we can derive the interpolation formula

$$
f(x+p h)=\sum_{n=0}^{\infty} a_{n} e^{-n x} P^{n} f(x)
$$

with

$$
\begin{aligned}
a_{0} & =1 \\
a_{n} & =\frac{\left(z^{p}-1\right)\left(z^{p-1}-1\right) \cdot \cdots\left(z^{p-n+1}-1\right)}{\left(1-z^{n}\right)\left(1-z^{n-1}\right) \cdot \cdot(1-z)} z^{n(n-1) / 2} .
\end{aligned}
$$

Again, (6.1) is most easily proven by applying it to the function $e^{-m x}$, $m=0,1 \cdots \infty$. This yields the identity

$$
\begin{aligned}
& z^{m p}=1+\sum_{n=1}^{\infty} \\
& \cdot \frac{\left(z^{p}-1\right)\left(z^{p-1}-1\right) \cdots\left(z^{p-n+1}-1\right) z^{n(n-1) / 2}\left(1-z^{m}\right)\left(1-z^{m-1}\right) \cdots\left(1-z^{m-n+1}\right)}{\left(1-z^{n}\right)\left(1-z^{n-1}\right) \cdots(1-z)} .
\end{aligned}
$$

The identity (6.3) is most easily proven for integral $m$ by induction on $m$, together with a straightforward direct proof for arbitrary $p$ when $m=0$.

All of the Eqs. (6.1), (5.1), (4.1) may be truncated after the $N$ th term; they are then exact when operating on a polynomial in $e^{-x}$ of degree $N$ or less. 
7. Integration Formulae. In this section we derive a number of integration rules, also designed to be exact when operating on polynomials in $e^{-x}$

(a) Rules derived from the interpolation formula (6.1).

We can derive a rule for the integral $I_{t, p}$

$$
I_{t, p} f(x)=\int_{t}^{t+p h} f(x) d x
$$

by integrating Eq. (6.1) for the function $f(t+q h)$ with respect to $q$ from 0 to $p$. In Eq. (7.1), $I_{t, p}$ is a linear operator acting on $f(x)$. If we cut off (6.1) after $N$ terms, the resulting rule is exact for polynomials in $e^{-x}$ of degree $N$; we can generate a sequence of rules of successively higher degree by increasing $N$. These rules are connected very simply with each other. If we write a rule of degree $N$ found in this way as

$$
I_{t, p}^{(N)} f(x)=\sum_{n=0}^{N} a_{n} e^{-n t} P^{n} f(t),
$$

then the annihilation property (3.5) implies that the coefficients $a_{n}$ are independent of $N$. We find on integration of (6.1) the following first few values for $a_{n}$ :

$$
\begin{aligned}
& a_{0}=p h, \\
& a_{1}=\frac{1-z^{p}-p h}{1-z}, \\
& a_{2}=\frac{-1+2 z(p h-1)+2 z^{p}+2 z^{p+1}-z^{2 p}}{2\left(1-z^{2}\right)(1-z)} .
\end{aligned}
$$

We have left the range of integration $p h$ free in Eq. (7.3). However, two particular choices of $p$ naturally stand out.

(i) An integration rule of degree $N$ of the form (7.2) contains the term $P^{N} f(t)$, and hence refers to the function $f$ evaluated at mesh points $x=t, t+h, \cdots, t+N h$. It is often convenient to refer only to points inside the range of integration, including the ends; we therefore take the particular choice $p=N$ and obtain the following rules from (7.3).

Rule A, Degree 1, $p=N=1$ :

$$
\int_{t}^{t+h} f(x) d x \approx\left[h+\frac{(1-z-h)}{1-z} e^{-t} P\right] f(t) .
$$

Rule B, Degree $2, p=N=2$ :

$$
\begin{aligned}
\int_{t}^{t+2 h} f(x) d x \approx[2 h & +\frac{1-z^{2}-2 h}{1-z} e^{-t} P \\
& \left.+\frac{-1+2 z(2 h-1)+2 z^{2}(1+z)-z^{4}}{2\left(1-z^{2}\right)(1-z)} e^{-2 t} P^{2}\right] f(t) .
\end{aligned}
$$

Rule $\mathrm{A}$ is exact for the functions $1, e^{-x}$, while rule $\mathrm{B}$ fits also $e^{-2 x}$. They are the analogues for the exponential functions of the usual trapezoidal rules and Simpson's rules respectively.

(ii) The choice of annihilation functions $e^{-m x}$ to be fitted suggests the construction of rules valid over an infinite domain $p \rightarrow \infty$. Such rules are useful in their own 
right for integrating exponentially decreasing functions over an infinite region, when the usual expansion in powers of $x$ of course runs into difficulties. The integration rule defined by (7.2), (7.3) is not useful for this purpose, since each of the terms diverges in this limit. This is a consequence of fitting the function 1.

(b) Modified rules. The results (7.3) are identical with the weights defined by insisting directly that the rule (7.2) fit the functions $1, e^{-x}, e^{-2 x}, \cdots$. We can obtain modified rules by not fitting the function 1 . The simplest of these is obtained by setting $a_{0}=0$ and writing

$$
\bar{I}_{t, p}^{(N)} f(x) \simeq \int_{t}^{t+p h} f(x) d x=\sum_{n=1}^{N} b_{n} e^{-n t} P^{n} f(t) .
$$

The coefficients $b_{n}$ in (7.6) are again independent of $N$, and the first two are

$$
\begin{aligned}
& b_{1}=\frac{1-z^{p}}{1-z} \\
& b_{2}=\frac{\left(1-z^{p}\right)\left(z^{p}-2 z-1\right)}{2(1-z)\left(1-z^{2}\right)} .
\end{aligned}
$$

The coefficients (7.7) do not diverge as $p \rightarrow \infty$; however, rule (7.6) is clearly not the most efficient possible. It is designed to fit the functions $e^{-m x}, m=1,2,3, \cdots$; but the degree 1 rule, involving two points, fits only one function $e^{-x}$, while the degree 2 rule obtained from (7.7) involves the evaluation of $f(x)$ at three points and fits only two functions.

A better procedure is therefore to write

$$
\bar{I}_{t, p}^{(N)} f(x) \simeq \int_{t}^{t+p} f(x) d x=\sum_{n=0}^{N} C_{n, N} e^{-n t} P^{n} f(t),
$$

where the coefficients $C_{n, N}$ now depend explicitly on the degree $N$, and are defined by the requirement that the rule of degree $N$ fit the functions $e^{-x}, e^{-2 x} \cdots e^{-(N+1) x}$. We find in this way the following rules.

Rule C, $N=1$, fits $e^{-x}, e^{-2 x}$

$$
\begin{aligned}
& C_{0,1}=\frac{\left(z^{p}-1\right)\left(z^{p}-2 z-1\right)}{2 z}, \\
& C_{1,1}=\frac{-\left(z^{p}-1\right)^{2}}{2 z(1-z)} .
\end{aligned}
$$

Rule $\mathrm{D}, N=2$, fits $e^{-x}, e^{-2 x}, e^{-3 x}$

$$
\begin{aligned}
& C_{0,2}=\frac{\left(1-z^{p}\right)}{6 z^{3}}\left\{6 z^{3}-\left(1-z^{p}\right)\left(1-3 z-3 z^{2}+2 z^{p}\right)\right\} \\
& C_{1,2}=\frac{\left(1-z^{p}\right)^{2}}{6 z^{3}(1-z)}\left(1-3 z-3 z^{2}+2 z^{p}\right) \\
& C_{2,2}=\frac{\left(1-z^{p}\right)^{2}\left(-1+3 z-2 z^{p}\right)}{6 z^{2}(1-z)\left(1-z^{2}\right)} .
\end{aligned}
$$

From these rules we obtain two rules of degree 1 and 2, analogous to (7.4) and $(7.5)$. 
Rule $\mathrm{C}^{\prime}, p=1, N=1$ :

$$
\int_{t}^{t+h} f(x) d x=\left[\frac{1-z^{2}}{2 z}+\frac{z-1}{2 z} e^{-t} P\right] f(t) .
$$

Rule $\mathrm{D}^{\prime}, p=2, N=2$ :

$$
\begin{aligned}
& \int_{t}^{t+2 h} f(x) d x=\left[\frac{\left(1-z^{2}\right)}{6 z^{3}}\left\{6 z^{3}-\left(1-z^{2}\right)\left(1-3 z-z^{2}\right)\right\}\right. \\
& \left.\quad+\frac{(1+z)^{2}(1-z)\left(1-3 z-z^{2}\right)}{6 z^{3}} e^{-t} P-\frac{\left(1-z^{2}\right)(1-2 z)}{6 z^{2}} e^{-2 t} P^{2}\right] f(t) .
\end{aligned}
$$

We also obtain two rules over the domain $t$ to $\infty$, valid for $h>0$.

Rule $\mathrm{C}^{\prime \prime}, N=1$ :

$$
\int_{t}^{\infty} f(x) d x=\left(\frac{1+2 z}{2 z}-\frac{1}{2 z(1-z)} e^{-t} P\right) f(t) .
$$

Rule $\mathrm{D}^{\prime \prime}, N=2$ :

$$
\begin{aligned}
\int_{t}^{\infty} f(x) d x=\left[\frac{-1+3 z+3 z^{2}+6 z^{3}}{6 z^{3}}\right. & +\frac{\left(1-3 z-3 z^{2}\right)}{6 z^{3}(1-z)} e^{-t} P \\
& \left.+\frac{(-1+3 z)}{6 z^{2}(1-z)\left(1-z^{2}\right)} e^{-2 t} P^{2}\right] f(t) .
\end{aligned}
$$

Rules $C^{\prime}, C^{\prime \prime}$ and $D^{\prime}, D^{\prime \prime}$ fit the same functions as rules $C, D$ respectively.

8. Numerical Examples. We conclude by giving some simple numerical examples of the use of the various rules derived above, and compare them with the corresponding conventional forward difference rules. We shall use the expansions on a number of simple test functions, which we define as follows

$$
\begin{aligned}
& f_{1}(x)=x+x^{2}+x^{3}, \\
& f_{2}(x)=e^{-x}+e^{-2 x}+e^{-3 x}, \\
& f_{3}(x)=x+x^{2}+x^{3}+x^{4} \\
& f_{4}(x)=e^{-x}+e^{-2 x}+e^{-3 x}+e^{-4 x}, \\
& f_{5}(x)=\sin x_{2} \\
& f_{6}(x)=x e^{-x^{2}} \\
& f_{7}(x)=x e^{-x} \\
& f_{8}(x)=x^{2} e^{-x} \\
& f_{9}(x)=1 /(1+x), \\
& f_{10}(x)=1 /\left(1+x^{2}\right) .
\end{aligned}
$$

The functions $f_{1}$ and $f_{2}$ are annihilated by $\Delta^{4}$ and $P^{4}$ respectively, and hence are fitted exactly with some of the rules tested below. The functions $f_{3}$ and $f_{4}$ test the 


\section{TABLE $\cdot \mathrm{I}$ \\ Interpolation \\ $h=0.1 \quad x=0.55$}

This table gives the results of retaining three and four terms in the interpolation formulae 6.1 and 9.2, for the functions (9.1). Column 2 gives the exact functional values, while columns 3 to 6 give the errors $\delta=f_{\text {approx. }}(x)-f(x)$ for the different approximations.

\begin{tabular}{|c|c|c|c|c|c|}
\hline Fu. No. & $f(x)$ exact & $p^{2}$ & $p^{3}$ & $\Delta^{2}$ & $\Delta^{3}$ \\
\hline 1 & 1.0188750 & $-0.0_{2} 1883$ & $+0.0_{3} 5311$ & $-0.0_{2} 375$ & 0 \\
\hline 2 & 1.1018708 & $+0.0_{4} 669$ & 0 & $+0.0_{3} 4822$ & $+0.0_{4} 681$ \\
\hline 3 & 1.1103813 & $-0.0_{2} 37175$ & $+0.0_{2} 12993$ & $-0.0_{2} 12563$ & +0.04937 \\
\hline 4 & 1.2126740 & $+0.0_{3} 2160$ & +0.0585 & $+0.0_{3} 8653$ & $\begin{array}{r}+0.0_{3} 1497 \\
\end{array}$ \\
\hline 5 & 0.5226872 & +0.04541 & -0.04260 & +0.04520 & $+0.0_{5} 23$ \\
\hline 6 & 0.4064327 & $+0.0_{3} 2828$ & +0.04107 & -0.04580 & +0.04505 \\
\hline 7 & 0.3173224 & $+0.0_{4} 360$ & -0.0547 & -0.08839 & $-0.0_{5} 70$ \\
\hline 8 & 0.1745273 & $+0.0_{4} 423$ & -0.04106 & +0.04982 & $+0.0_{4} 154$ \\
\hline 9 & 0.6451613 & +0.04155 & $-0.0_{6} 8$ & +0.04593 & $+0.0_{5} 82$ \\
\hline 10 & 0.7677543 & $-0.0_{3} 1034$ & $+0.05_{5} 99$ & $-0.0_{3} 1763$ & $-0.0 \_378$ \\
\hline
\end{tabular}

\section{TABLE 2 \\ Differentiation \\ $h=0.1 \quad x=0$}

This table gives the results of retaining three and four terms in the differentiation formulae (5.2) and (9.3) for the functions (9.1). Column 2 gives the exact derivative $f^{\prime}(0)$, while columns 3 to 6 give the errors $\delta=f_{\text {approx. }}^{\prime}(0)-f^{\prime}(0)$ for the different approximations.

\begin{tabular}{|c|c|c|c|c|c|}
\hline Fu. No. & $f^{\prime}(x)$ & $p^{2}$ & $p^{3}$ & $\Delta^{2}$ & $\Delta^{3}$ \\
\hline 1 & 1.0 & -0.0564945 & +0.221590 & -0.02 & 0 \\
\hline 2 & -6.0 & -0.0172501 & 0 & -0.0984157 & -0.0176536 \\
\hline 3 & 1.0 & -0.0640372 & +0.0353901 & -0.026 & +0.006 \\
\hline 4 & -10.0 & -0.0814818 & $-0.0_{2} 44709$ & -0.2581718 & -0.0579679 \\
\hline 5 & 1.0 & $-0.0_{2} 27582$ & $-0.0_{3} 3684$ & $+0.0_{2} 33217$ & $+0.0_{4} 299$ \\
\hline 6 & 1.0 & +0.0186146 & $-0.0_{2} 84117$ & +0.0193102 & $+0.0_{2} 17124$ \\
\hline 7 & 1.0 & $+0.0_{2} 33311$ & -0.035304 & $-0.0_{2} 90559$ & $-0.0_{3} 8618$ \\
\hline 8 & 0.0 & $+0.0_{3} 4996$ & -0.036589 & +0.0172213 & $+0.0_{2} 24582$ \\
\hline 9 & -1.0 & $+0.0_{2} 50653$ & $+0.00_{3} 5013$ & +0.0151515 & $+0.0_{2} 34965$ \\
\hline 10 & 0.0 & +0.0154661 & $+0.0_{2} 39245$ & $-0.0_{2} 57121$ & +0.0246640 \\
\hline
\end{tabular}

degree to which one extra term affects the accuracy of the result; while the functions $f_{5}$ to $f_{10}$ were chosen merely as examples of a variety of well-behaved functions.

A. Interpolation. The comparable forward difference interpolation formula to (6.1) is given by

$$
\begin{aligned}
f(x+p h) & =\sum_{n=0}^{\infty} b_{n} \Delta^{n} f(x) \\
b_{0} & =1 \\
b_{n} & =\frac{p(p-1) \cdots(p-n+1)}{n !} .
\end{aligned}
$$




\section{TABLE 3 \\ Integration}

Integration between zero and two, $h=0.1$

This table compares the two exponential-Simpson integration rules 7.5 and 7.12 with the usual Simpson's rule. Column 2 gives the exact result, while columns 3 to 5 give the error $\int_{0}^{2} f(x) d x$ (approx.) - $\int_{0}^{2} f(x) d x$, for these different rules.

\begin{tabular}{c|c|c|c|c}
\hline Fu. No. & $\int_{0}^{2} f(x) d x$ exact & Eq. 7.5 & Eq. 7.12 & Simpson \\
\cline { 2 - 3 } & 8.6666667 & $-0.0_{3} 1089$ & $-0.0_{3} 8662$ & 0 \\
2 & 1.6880140 & $+0.0_{5} 66$ & 0 & $+0.0_{4} 196$ \\
3 & 15.066667 & $-0.0_{3} 260$ & $-0.0_{2} 1951$ & $+0.0_{4} 26$ \\
4 & 1.9379301 & $+0.0_{4} 294$ & $+0.0_{5} 82$ & $+0.0_{4} 545$ \\
5 & 1.4161468 & $+0.0_{5} 33$ & $-0.0_{4} 382$ & $+0_{6} 8$ \\
6 & 0.4908422 & $+0.0_{5} 73$ & $+0.0_{5} 77$ & $+0.0_{5} 30$ \\
7 & 0.5939942 & $+0.0_{5} 19$ & $-0.0_{5} 68$ & $-0.0_{5} 16$ \\
8 & 0.6466472 & $+0.0_{5} 17$ & $-0.0_{4} 276$ & $+0_{5} 34$ \\
9 & 1.0986123 & $+0.0_{5} 20$ & $-0.0_{5} 89$ & $+0.0_{5} 32$ \\
10 & 1.1071487 & $+0.0_{5} 32$ & $+0.0_{5} 67$ & $-0.0_{6} 1$ \\
\hline
\end{tabular}

\section{TABLE 4}

Integration over an infinite range

This table gives the results of using Eq. (7.14) to estimate the integral $\int_{a}^{\infty} f(x) d x$, for various lower limits $a$ and step length $h$. Columns 3,4 and 6 give the deviations of this rule from the exact values.

\begin{tabular}{c|c|c|c|c|c}
\hline Fu. No. & $\int_{2}^{\infty} f(x) d x$ & $\begin{array}{c}\text { Eq. (7.14), } \\
h=0.1\end{array}$ & $\begin{array}{c}\text { Eq. (7.14), } \\
h=0.5\end{array}$ & $\int_{3}^{\infty} f(x) d x$ & $\begin{array}{c}\text { Eq. (7.14), } \\
h=0.1\end{array}$ \\
\cline { 2 - 5 } 2 & 0.1453193 & \multicolumn{1}{c}{0} & 0 & 0.0510675 & 0 \\
4 & 0.1454032 & +0.04558 & +0.04136 & 0.0510691 & $+0.0_{5} 10$ \\
6 & 0.0091578 & $-0.0_{2} 47620$ & $+0.0_{3} 4901$ & 0.04617 & $+0.0_{3} 247$ \\
7 & 0.4060058 & -0.0406499 & -0.0252380 & 0.1991483 & -0.0149543 \\
8 & 1.3533528 & -0.3742488 & -0.2553019 & 0.8463802 & -0.1675871 \\
10 & 0.4636476 & -0.2230943 & -0.2036500 & 0.3217505 & -0.1864093 \\
\hline
\end{tabular}

Table 1 gives the accuracy attained by retaining second and third order differences in Eqs. (8.2) and (6.1), for the functions $f_{1}-f_{10}$.

B. Differentiation. The comparable forward difference formula to (5.2) is given by

$$
D=\sum_{n=1}^{\infty}(-1)^{n+1} \frac{\Delta^{n}}{n h} .
$$

Table 2 gives a comparison of (8.3) and (5.2).

C. Integration. The integration rules of Section 7 share one useful property with the usual rules fitted to polynomials in $x$ : they are translationally-invariant, that is, the relative weights $a_{n}$ assigned to the points in basic cell are independent of the origin of the cell. It is therefore a simple matter to generate cytolic rules over a large region by piecing together rules for smaller regions.

Table 3 compares such cytolic rules generated from the three point formulae (7.5) and (7.12), with the usual cytolic Simpson's rule, for the region $0 \leqq x \leqq 2$. In this table, as in Tables 1 and 2, the step length is taken to be $h=0.1$. 
Finally, Table 4 gives an example of integration over an infinite domain using Eq. (7.14).

9. Discussion. These illustrative examples show clearly the expected result: expansions in $P$ are better than expansions in $\Delta$ for some functions, and worse for others. The necessary exponential differences are more time-consuming to form than the usual difference tables; but the gain in their use is, for suitable functions, so marked as to more than compensate for this. This is especially so if, as may well occur in practice, the calculation of the functions themselves takes much longer than the differencing.

The results of Tables 3 and 4 are especially interesting. We see that, except in the special case of function 2, the rule (7.5) is better than (7.12). We recall that, although both rules contain terms up to $P^{2}$, Eq. (7.5) fits the function $1, e^{-x} e^{-2 x}$, while (7.12) fits the function $e^{-3 x}$ but not the function 1. For a cytolic numerical integration scheme, in which the whole region of integration is divided into unit cells which are integrated over separately, it is clearly usually (but again not always) advantageous to fit the constant function.

The major justification of (7.12) is that it leads to the rule (7.14) for integration over an infinite region. Table 4 shows clearly the use of the rule in estimating the tail of an integral. It also shows, as should be expected, that it does not pay to make $h$ small in this estimation.

We have discussed in this paper only forward differences, but backward and central differences formulae can be given in a similar manner; and the simple results given here can be extended in a number of directions in a straightforward manner. Some of these extensions will be dealt with in later papers.

Acknowledgement. I am grateful to Dr. J. N. Lyness and Mr. C. Cox for useful discussions of this work, most of which was carried out while the author was at the University of New South Wales. * 5287.

1. H. Levy \& F. Lessmann, Finite Difference Equations, Pitman, London, 1958. MR 21

2. S. Montmort, Philos. Trans. Roy. Soc. London, 1717; see [1, p. 27].

3. R. E. Greenwood, "Numerical integration for linear sums of exponential functions," Ann. Math. Statist., v. 20, 1949, pp. 608-611. MR 11, 266.

4. P. Brock \& F. J. Murray, "The use of exponential sums in step by step integration," $M T A C$, v. 6,1952 , pp. $63-78$. MR $13,873$.

5. H. W. Gound, "The operator $\left(a^{x} \Delta\right)^{n}$ and Stirling numbers of the first kind," Amer. Math. Monthly, v. 71, 1964, p. 850 . 